Proyecciones Journal of Mathematics

Vol. 31, No 1, pp. 81-90, March 2012.

Universidad Católica del Norte

Antofagasta - Chile

\title{
On an algorithm for finding derivations of Lie algebras *
}

\author{
VÍCTOR AYALA \\ Universidad Católica del Norte, Chile \\ EYÜP KIZIL \\ Universidade de Sao Paulo, Brasil \\ IVAN DE AZEVEDO TRIBUZY \\ Universidade Federal de Amazonas, Brasil \\ Received : May 2011. Accepted : December 2011
}

\begin{abstract}
Let $\mathbf{g}$ be an arbitrary finite dimensional Lie algebra over the field R. We give as an additional alternative a detailed overview of an algorithm for finding derivations of $\mathbf{g}$ since such procedures are often of interest.
\end{abstract}

AMS classification : 16W25; 93B29; 93805

Key words : Derivations of Lie algebras; Linear control system; Null controllability

${ }^{*}$ Research partially supported by Conicyt Proyecto Fondecyt N 1100375 


\section{Introduction}

All the necessary theoretical foundations for Lie algebras and results of their algebraic characteristics in case of low-dimensions are well known. In particular, derivations of Lie algebras are frequently considered in the literature, [4], [7], etc. On the other hand, the theoretical method to compute derivations is obvious while their effective computation is not an easy task. For this reason computer programs for calculation of Lie algebra characteristics such as automorphisms, ideals, derivations, etc are in use for a long time.

In the computer algebra system $\mathrm{GAP}^{1}$ it can be found a procedure to compute derivations of Lie algebras defined either over finite fields (with some restrictions) and over fields of char 0 , but only for rationals. That is, it does not compute with Lie algebras having pure real or complex non-rational structure constants. Moreover, the program does not support parameters.

With Matematica, there are also some procedures to compute derivations with reasonably limited applications since none of them has been conceived to consider parameters.

In this paper we consider the structure tensor to obtain conditions that a linear transformation must satisfy in order to be a derivation of a Lie algebra over the field of reals. This, of course, leads to a case by case analysis whenever we have parameters.

\section{Derivations of Lie algebras}

Let $\mathbf{g}$ be an $n$ dimensional real Lie algebra and take a basis $\left\{X_{1}, \ldots, X_{n}\right\}$ for $\mathbf{g}$. By bilinearity, the bracket operation $[\cdot, \cdot]$ in $\mathbf{g}$ is completely determined once the values $\left[X_{i}, X_{j}\right], 1 \leq i, j \leq n$ are known. With the Einstein notation, the coefficients $c_{i j}^{k} \in \mathbf{R}, 1 \leq i, j, k \leq n$, in $\left[X_{i}, X_{j}\right]=c_{i j}^{k} X_{k}$ (sum over $k$ ) are uniquely determined and called the structure constants of $\mathbf{g}$ relative to the given basis. Then it follows that brackets of any two elements can be obtained by using the $n^{3}$ constants $c_{i j}^{k}$. Also, a set of structure constants satisfies:

$$
\begin{aligned}
c_{i j}^{k} & =-c_{j i}^{k}, \quad 1 \leq i, j, k \leq n, \\
c_{i l}^{m} c_{j k}^{l}+c_{j l}^{m} c_{k i}^{l}+c_{k l}^{m} c_{i j}^{l} & =0, \quad 1 \leq i, j, k, l, m \leq n .
\end{aligned}
$$

\footnotetext{
${ }^{1}$ For further information on GAP, see www.gap-system.org
} 
Definition 2.1. A linear transformation $D: \mathbf{g} \rightarrow \mathbf{g}$ is said to be a derivation of $\mathbf{g}$ if it satisfies

$$
D([X, Y])=[D(X), Y]+[X, D(Y)] \text { for all } X, Y \text { in } \mathbf{g} .
$$

The matrix representation of $D$ with respect to the basis above is the $n \times$ $n$ matrix $[D]=\left(d_{i j}\right)^{T}$ whose entries are defined by the equations $D\left(X_{i}\right)=$ $\sum_{p=1}^{n} d_{i p} X_{p}, 1 \leq i \leq n$. Given a Lie algebra $\mathbf{g}$, we denote by $\operatorname{Der}(\mathbf{g})$ the Lie algebra of all derivations of $\mathbf{g}$.

It is natural to divide the problem of computing derivations of a Lie algebra $\mathbf{g}$ into two parts, namely,

1. Computing the inner derivations $\operatorname{ad}(\mathbf{g})=\mathbf{g} / \mathcal{C}(\mathbf{g})$ where ad means the adjoint representation of $\mathbf{g}$ and here a basis of the center $\mathcal{C}(\mathbf{g})$ of $\mathbf{g}$ can be read off immediately from the structure constants,

2. Computing the outer derivations $\operatorname{Der}(\mathbf{g}) / \operatorname{ad}(\mathbf{g})$.

Of course, these can be expressed as a cohomology space $\mathcal{H}^{1}(\mathbf{g}, \mathbf{g})$ for which several algorithms exist in the literature. See, for example, the survey paper by D. Leites and G. Post, [5]. For instance, if we consider Heisenberg Lie algebra $\mathbf{g}$ of dimension 3 generated by the vector fields

$$
X_{1}=\frac{\partial}{\partial x_{1}} \quad X_{2}=\frac{\partial}{\partial x_{2}}+x_{1} \frac{\partial}{\partial x_{3}} \quad X_{3}=\frac{\partial}{\partial x_{3}}
$$

with the only non-vanishing Lie bracket $\left[X_{1}, X_{2}\right]=X_{3}$ it follows that we obtain $\mathcal{H}^{1}(\mathbf{g}, \mathbf{g})=4$ from which it follows immediately by the procedure sketched above that $\operatorname{dim} \operatorname{Der}(\mathbf{g})=6$.

\subsection{Outline of the derivation algorithm}

A rough description or idea of an algorithm for finding derivations may be found in Kolman-Beck, [2], but we find it convenient to provide a detailed overview of this algorithm since such procedures are often of interest. We suggest [3] as a reference for both theoretical and algorithmical aspects of Lie algebras.

It is clear that in order to determine derivations of $\mathbf{g}$ it is enough to verify the condition in (2.1) only for brackets $\left[X_{i}, X_{j}\right]$ between basis elements of $\mathbf{g}$. On the other hand, since we are concerned with an algorithm that calculate with Lie algebras we need to represent Lie algebras in such a way that they can be dealt with by a computer, that is, as lists of numbers. Hence, we take into account structure constants of $\mathbf{g}$ and express below the condition (2.1) in terms of these numbers. 
Proposition 2.2. Let $\mathbf{g}$ be a $n$-dimensional real Lie algebra and fix a basis $\left\{X_{1}, X_{2}, \ldots, X_{n}\right\}$ for it. Let $D$ denote a linear transformation on $\mathbf{g}$ whose matrix relative to this basis is $\left(d_{i j}\right)^{T}$. Then $D$ is a derivation of $\mathbf{g}$ if and only if

$$
\sum_{k=1}^{n} c_{i j}^{k} d_{k p}=\sum_{k=1}^{n}\left(d_{i k} c_{k j}^{p}+d_{j k} c_{i k}^{p}\right)
$$

for every $1 \leq i, j, p \leq n$.

The preceding proposition simply says that a derivation is a solution of the homogeneous system (2.2) consisting of $n^{3}$ linear equations for the $n^{2}$ unknowns $d_{i j}$.

For computer purposes a $n \times n^{2}$ matrix $A=\left(a_{i j}\right)$ whose entries are the structure constants of $\mathbf{g}$ is needed. Actually, since $c_{i i}^{p}=0,1 \leq i, p \leq n$, the matrix $A$ has the following face:

$$
\left(\begin{array}{lllllllllllllll} 
& 0_{n} & & & & c_{1 j}^{1} & c_{1 j}^{2} & \ldots & c_{1 j}^{n} & \ldots & & & & \\
& & & & & c_{2 j}^{1} & c_{2 j}^{2} & \ldots & c_{2 j}^{n} & \ldots & & & & \\
& & & & & : & : & & : & & & & & \\
c_{i 1}^{1} & c_{i 1}^{2} & \ldots & c_{i 1}^{n} & \ldots & c_{i j}^{1} & c_{i j}^{2} & \ldots & c_{i j}^{n} & \ldots & c_{i n}^{1} & c_{i n}^{2} & \ldots & c_{i n}^{n} \\
& & & & & : & : & & : & & & & & \\
& & & & & c_{n j}^{1} & c_{n j}^{2} & \ldots & c_{n j}^{n} & \ldots & & & 0_{n} &
\end{array}\right)
$$

where $0_{n}$ denotes the zero sequence $(0,0, \ldots, 0)$.

It follows that the structure constants $c_{i j}^{k}$ on the left side of (2.2) correspond to $i,(j-1) n+k$ th entries of $A$. Hence, the sum over $k$ corresponds to vector multiplication of $p$ th column $(1 \leq p \leq n)$ of the matrix $\left(d_{i j}\right)$ with $\left(a_{i, j n-n+1}, a_{i, j n-n+2}, \ldots, a_{i, j n}\right)$.

Analogously, the sum $\sum_{k=1}^{n} d_{i k} c_{k j}^{p}$ means the multiplication of the $i$ th row of $\left(d_{i j}\right)$ with $j n-n+p$ th column of the matrix $A$.

Finally, $\sum_{k=1}^{n} d_{j k} c_{i k}^{p}$ states the multiplication of $j$ th row of $\left(d_{i j}\right)$ with the vector $\left(c_{i 1}^{p} c_{i 2}^{p} \ldots c_{i n}^{p}\right), 1 \leq p \leq n$.

If we have a look at the $i$ th row of the matrix $A$ it becomes clear that for each $p$, coordinates of the vector $\left(c_{i 1}^{p} c_{i 2}^{p} \ldots c_{i n}^{p}\right)$ are obtained through the entries of $A$ as follows: Starting from the $p$ th column of $A$ we skip $n$ column each time and pick the entry of $A$ located therein. This implies that $c_{i k}^{p}$ corresponds to $i,(k-1) n+p$ th entry of $A$, and hence

$$
\left(\begin{array}{cccc}
c_{i 1}^{p} & c_{i 2}^{p} & \ldots & c_{i n}^{p}
\end{array}\right)=\left(\begin{array}{llll}
a_{i, p} & a_{i, n+p} & \ldots & a_{i, n^{2}-n+p}
\end{array}\right) .
$$


It follows that $\sum_{k=1}^{n} d_{j k} c_{i k}^{p}$ is the same as multiplication of $j$ th row of $\left(d_{i j}\right)$ with $\left(a_{i, p} a_{i, n+p} \ldots a_{i, n^{2}-n+p}\right)$.

Remark: Those structure constants for which $i \geq j$ can even be deduced from the others due to reflexivity and anti-symmetry property of Lie parenthesis $[$,$] . Therefore, we are allowed to reduce the number of equations in$ (2.2) and consider only $n^{2}(n-1) / 2$ equations instead of $n^{3}$.

Let $l$ denotes the left side of (2.2) while the sum $r 1+r 2$ stands for the right side. Then a formal description of derivation algorithm may be given in the following way.

Step1. Input the dimension $n$

Step2. Define an $n \times n$ matrix $\left(d_{i j}\right)$

Step3. Input the $n \times n^{2}$ matrix $A=\left(a_{i j}\right)$

Step4. Let $i=1,2, \ldots, n-1, \quad j=i+1, i+2, \ldots, n$, and $1 \leq p, k \leq n$.

Determine the sums

$$
\begin{aligned}
l & =\sum_{k=1}^{n} d_{k p} a_{i,(j-1) n+k} \\
r 1 & =\sum_{k=1}^{n} d_{i k} a_{k,(j-1) n+p} \\
r 2 & =\sum_{k=1}^{n} d_{j k} a_{i,(k-1) n+p}
\end{aligned}
$$

and display ( $n$-times) $l$ and $r 1+r 2$ for each $(i, j)$.

Apart from this description it becomes easy to establish, for example, a maple code for such an algorithm. We left this part to interested users. Just observe that the printout of the above algorithm lists for each $(i, j)$ a line of type $l=r 1+r 2$ and hence $n^{2}(n-1) / 2$ equations in total appearing in $(2.2)$.

\section{Examples}

1. The orthogonal Lie algebra $\mathrm{o}(3)$.

Let $X_{1}, X_{2}$ and $X_{3}$ denote three infinitesimal rotations around the $x$, $y$ and $z$-axis. They are a basis for o $(3), 3 \times 3$ real skew matrices. One computes $\left[X_{1}, X_{2}\right]=X_{3},\left[X_{2}, X_{3}\right]=X_{1}$ and $\left[X_{3}, X_{1}\right]=X_{2}$. Hence the non-vanishing structure constants are

$$
c_{12}^{3}=c_{23}^{1}=c_{31}^{2}=1
$$


and derivations of o(3) can be obtained through the matrix

$$
A=\left(\begin{array}{ccccccccc}
0 & 0 & 0 & 0 & 0 & 1 & 0 & -1 & 0 \\
0 & 0 & -1 & 0 & 0 & 0 & 1 & 0 & 0 \\
0 & 1 & 0 & -1 & 0 & 0 & 0 & 0 & 0
\end{array}\right)
$$

which gives us the following table

\begin{tabular}{|l|l|l|}
\hline \multicolumn{1}{|c|}{$(i, j)=(1,2)$} & \multicolumn{1}{|c|}{$(i, j)=(1,3)$} & \multicolumn{1}{c|}{$(i, j)=(2,3)$} \\
\hline$d_{31}=-d_{13}$ & $-d_{21}=d_{12}$ & $d_{11}=d_{22}+d_{33}$ \\
$d_{32}=-d_{23}$ & $-d_{22}=-d_{11}-d_{33}$ & $d_{12}=-d_{21}$ \\
$d_{33}=d_{11}+d_{22}$ & $-d_{23}=d_{32}$ & $d_{13}=-d_{31}$ \\
\hline
\end{tabular}

This table contains inside the following homogeneous system

$$
\begin{array}{r}
d_{11}+d_{22}-d_{33}=0 \\
-d_{11}+d_{22}-d_{33}=0 \\
d_{11}-d_{22}-d_{33}=0
\end{array}
$$

whose matrix of coefficients is invertible. Thus, it has only trivial solution resulting that $d_{11}=d_{22}=d_{33}=0$ while $d_{21}=-d_{12}, d_{31}=$ $-d_{13}$ and $d_{32}=-d_{23}$. Therefore, a derivation of the orthogonal Lie algebra has the following face

$$
D=\left(\begin{array}{ccc}
0 & z & y \\
-z & 0 & x \\
-y & -x & 0
\end{array}\right)
$$

Working out the adjoint representation one obtains $D_{1}=\operatorname{ad}\left(X_{1}\right)$, $D_{2}=\operatorname{ad}\left(X_{2}\right)$ and $D_{3}=\operatorname{ad}\left(X_{3}\right)$. This means that any derivation of o(3) is inner, a general fact that occurs for semi-simple Lie algebras. Hence we have just seen that this fact confirms the printout of algorithm.

2. The Heisenberg Lie algebra of dimension 3.

Let $\mathbf{g}$ denote the Heisenberg Lie algebra generated by the vector fields $X_{1}=\frac{\partial}{\partial x_{1}}, X_{2}=\frac{\partial}{\partial x_{2}}+x_{1} \frac{\partial}{\partial x_{3}}$ and $X_{3}=\frac{\partial}{\partial x_{3}}$. The only non empty Lie bracket is $\left[X_{1}, X_{2}\right]=X_{3}$. Hence one has the relations listed as follows:

\begin{tabular}{|l|c|c|}
\hline$(i, j)=(1,2)$ & $(i, j)=(1,3)$ & $(i, j)=(2,3)$ \\
\hline$d_{31}=0$ & $0=0$ & $0=0$ \\
$d_{32}=0$ & $0=0$ & $0=0$ \\
$d_{33}=d_{11}+d_{22}$ & $0=d_{32}$ & $0=-d_{31}$ \\
\hline
\end{tabular}


A simple algebraic manipulation shows that a derivation $D$ of $\mathbf{g}$ in its matrix form is given by

$$
[D]=\left(\begin{array}{ccc}
x & y & 0 \\
z & t & 0 \\
u & v & x+t
\end{array}\right) .
$$

Since we have only six different parameters it follows at once that the dimension of the Lie algebra $\operatorname{Der}(\mathbf{g})$ is equal to 6 .

Remark: We note that the Lie algebras considered in examples 1 and 2 are such that any nonvanishing Lie bracket is one of the algebra generators. This lets the printout of the algorithm much easier to determine a basis for the Lie algebra of derivations.

Next, we consider an example of a Lie algebra admitting also Lie brackets as linear combination of its generators.

3. Let $\mathbf{g}$ be the Lie algebra of dimension 4 with the bracket rules

$$
\begin{aligned}
& {\left[X_{4}, X_{2}\right]=X_{4}} \\
& {\left[X_{3}, X_{2}\right]=X_{4}+X_{3}} \\
& {\left[X_{1}, X_{i}\right]=X_{i+1} \text { for } i=2,3}
\end{aligned}
$$

Therefore we have a $4 \times 16$ matrix $A$ that yields the following relations:

\begin{tabular}{|l|l|}
\hline$(i, j)=(1,2)$ & $(i, j)=(1,3)$ \\
\hline$d_{31}=0$ & $d_{41}=0$ \\
$d_{32}=0$ & $d_{42}=0$ \\
$d_{33}=d_{11}+d_{13}+d_{22}$ & $d_{43}=-d_{12}+d_{32}$ \\
$d_{34}=d_{13}+d_{14}+d_{23}$ & $d_{44}=d_{11}-d_{12}+d_{33}$ \\
\hline$(i, j)=(1,4)$ & $(i, j)=(2,3)$ \\
\hline $0=0$ & $-d_{31}-d_{41}=0$ \\
$0=0$ & $-d_{32}-d_{42}=0$ \\
$0=d_{42}$ & $-d_{33}-d_{43}=-d_{22}-d_{31}-d_{33}$ \\
$0=-d_{12}+d_{43}$ & $-d_{34}-d_{44}=d_{21}-d_{22}-d_{33}-d_{34}$ \\
\hline$(i, j)=(2,4)$ & $(i, j)=(3,4)$ \\
\hline$-d_{41}=0$ & $0=0$ \\
$-d_{42}=0$ & $0=0$ \\
$-d_{43}=-d_{41}-d_{43}$ & $0=0$ \\
$-d_{44}=-d_{22}-d_{43}-d_{44}$ & $0=-d_{32}-d_{41}+d_{42}$ \\
\hline
\end{tabular}


A simple computation shows that a derivation $D$ has the following matrix

$$
[D]=\left(\begin{array}{cccc}
x & -x & 0 & 0 \\
0 & 0 & 0 & 0 \\
y & t & x+y & 0 \\
z & u & y+z+t & 2 x+y
\end{array}\right)
$$

and hence $\operatorname{Der}(\mathbf{g})$ is a 5 dimensional Lie algebra.

\section{Final comments}

The above algorithm is interesting because it gives the relations and thus one can easily look for a basis. It would also be helpful, for example, for control systems on Lie groups as follows.

1. Derivations of Lie algebras together with an algorithm to facilitate their efficient computation may be a quite useful tool for linear control systems on Lie groups introduced by Ayala-Tirao in [1]. More precisely, for a connected Lie group $G$ the notion of a linear control system

$$
\Sigma=(G, \mathcal{D})
$$

where $G$ is the state space and $\mathcal{D}$ is the dynamic given by a family of differential equations parametrized by control functions has its drift vector field $X$ as an element of the normalizer

$$
n=\left\{Z \in \operatorname{Vect}^{\infty}(G):[Z, \mathbf{g}] \subset \mathbf{g}\right\}
$$

of $\mathbf{g}$ in the set of smooth vector fields $\operatorname{Vect}^{\infty}(G)$ of $G$. Here $\mathbf{g}$ is considered as the set of left invariant vector fields. It turns out that every element of $\operatorname{Der}(\mathbf{g})$ induces an element $X \in n$. In fact, the authors prove in [1] that

$$
n=\mathbf{g} \times{ }_{s} \operatorname{Der}(\mathbf{g})
$$

where $\times_{s}$ stands for semi-direct product. On the other hand, by considering one parameter groups of inner automorphisms on $G$ it is very easy to construct a subclass of drift vector fields in $\operatorname{Der}(\mathbf{g})$, which are related to the adjoint representation of the Lie algebra $\mathbf{g}$. However, we stress that this subclass is far from determining Der $(\mathbf{g})$. Moreover, it is given in [1] an explicit formula for trajectories of $\Sigma$ in which derivations of $\mathbf{g}$ defining $X$ appear. In general, it is hard to compute these trajectories but having concrete derivations at hand would make this difficult task become relatively easier. 
2. Derivations can also help to determine local controllability. Indeed, according to Theorem 3,5 in [1] any (transitive) linear control system $\Sigma$ on a connected Lie group $G$ with Lie algebra $\mathbf{g}$ is locally controllable if it satisfies ad-rank condition

$$
\operatorname{dim}\left\{\operatorname{Span}\left\{Y^{j}, \operatorname{ad}^{k}(X)\left(Y^{j}\right): 1 \leq k \leq p, 1 \leq j \leq m\right\}=\operatorname{dim}(G)\right.
$$

where $p$ is an integer such that $0<p<\operatorname{dim}(G)$.

Since $\operatorname{ad}(X)(Y)=D(Y)$ where $D \in \operatorname{Der}(\mathbf{g})$ it follows that one can determine explicitly ad-rank sequence just by matrix multiplication with consecutive iterations.

3. A linear control system $\Sigma$ on a connected Lie group $G$ is said to be null controllable if for each $g \in G$ there exists a control function $u \in \mathcal{U}$ and $t>0$ such that the integral curve $x(g, u, t)=e \in G$, the identity element. When $G$ is a connected and simply connected nilpotent Lie group, it is known that the exponential map $\exp _{G}: \mathrm{g} \rightarrow G$ is an isomorphism from the Abelian Lie group $\mathrm{g}$ onto $G$. It seems natural to us that local controllability from $e \in G$ together with some spectrum condition on derivations (for example, derivations whose eigenvalues have negative real part) could provide globally null controllable systems on this particular class of Lie groups.

\section{References}

[1] V. Ayala and J. Tirao, Linear control systems on Lie groups and controllability, in:Proceedings of the American Mathematical Society, Series: Symposia in Pure Mathematics, Vol. 64, (1999).

[2] E. Beck, B. Kolman and I.N. Stewart, Computing the structure of a Lie algebra, in:R.E.Beck and B. Kolman, editors, Non-associative rings and algebras, Academic press, pp. 167-188, (1977).

[3] W. A. de Graaf, Lie algebras: Theory and Algorithms, North-Holland Mathematical Library, (2000).

[4] G. F. Leger, A note on the derivations of Lie algebras, Proc. Amer. Math. Soc. 4, pp. 511-514, (1953). 
[5] D. Leites and G. Post, Cohomology to compute, Proceedings of the third conference on Computers and Mathematics, pp. 73-81, (1989).

[6] A. O. Nielsen, Unitary representations and coadjoint orbits of low dimensional nilpotent Lie groups, Queen's Papers in Pure and Applied Mathematics 63, (1983).

[7] S. Tôgô, Derivations of Lie algebras. J. Sci. Hiroshima Univ. Ser. A-1Math 28pp. 133-158, (1964).

[8] V.S. Varadaradjan, Lie groups, Lie algebras and Their representations, Prentice-Hall, (1974).

\section{Victor Ayala}

Departamento de Matemáticas

Universidad Católica del Norte

Casilla 1280,

Antofagasta,

Chile

e-mail : vayala@ucn.cl

\section{Eyüp Kizil}

Instituto de Ciências Matemáticas e de Computação.

Universidade de São Paulo.

Cx. Postal 668. 13.560-970,

São Carlos-SP,

Brasil

e-mail : kizil@icmc.usp.br

and

Ivan de Azevedo Tribuzy

Instituto de Ciências Exatas.

Universidade Federal de Amazonas.

Manaus,

Brasil

e-mail : ivan@argo.com.br 